\title{
Structures of TAPBPR/MHC-I and TAPBPR/nanobody complexes; rigidification of dynamic regions on interaction with ligands
}

\author{
Jiansheng Jiang, Kannan Natarajan, David H. Margulies \\ jiangji@niaid.nih.gov knatarajan@niaid.nih.gov dmargulies@niaid.nih.gov \\ Molecular Biology Section, Laboratory of Immune System Biology, National Institute of Allergy and \\ Infectious Diseases, NIH, Bethesda, MD, 10892-1892 \\ Structures of the tapasin homolog, TAP binding protein, related (TAPBPR), complexed with major \\ histocompatibility complex class I (MHC-I) molecules, revealed conformational changes of MHC-I with \\ peptide loading, contributing to a mechanistic understanding of peptide editing in antigen presentation \\ $[1,2]$. In an effort to study the dynamic flexibility of TAPBPR, we isolated TAPBPR-specific nanobodies \\ (camelid-derived single domain antibody fragments), from phage display libraries generated from \\ peripheral blood lymphocyte mRNA of an alpaca immunized with TAPBPR. E. coli-expressed and purified \\ nanobodies bound tightly to TAPBPR and the crystal structure of a TAPBPR/nanobody complex was \\ determined. The nanobody binds to the IgC domain of TAPBPR distant from the MHC-I binding site \\ which also involves the $\mathrm{N}$-terminal domains. Nanobody binding rigidifies the region of contact \\ permitting modeling of TAPBPR residues that were not visible in the electron density map of the \\ TAPBPR/MHC-I complex. Conversely, the MHC-I binding site on TAPBPR is more disordered in the \\ nanobody complex. Additionally, the hinge angle between the $\mathrm{N}$-terminal and IgC domains of TAPBPR \\ differs in the two complexes. These data are consistent with the view that the domains of TAPBPR, in \\ solution, are structurally dynamic and rigidify on ligand (i.e. MHC-I) interaction. Such structural plasticity \\ may explain the ability of TAPBPR to stabilize many different MHC-I molecules, and to optimize the \\ selection of high affinity peptides during antigen presentation.
}

References:

[1] Jiang, et al., Science 2017, 358, 1064-68.

[2] Thomas, et al., Science 2017, 358, 1060-64.

(Supported by the Intramural research program of the NIAID, NIH) 